\title{
Compaction Behavior and Mechanical Properties of Uniaxially Pressed Bi-W Composites
}

\section{PETER MARTIN, ANDREA M. HODGE, and GEOFFREY H. CAMPBELL}

Powder metallurgy is a useful route to forming particulate composite materials; however, the densification of hard and soft powder mixtures is usually inhibited by the more refractory phase. The Bi-W powder compacts were uniaxially pressed at room temperature and the compaction behavior and mechanical properties were evaluated. Pressing was performed in incremental steps from $\sim 1$ to $540 \mathrm{MPa}$. After each step, the pressure was relieved and the thickness and sound-wave transit time were measured in situ (in the die), in order to determine the density and sound-wave velocity in the compact. The data show that the unreinforced Bi powder compacts to $\sim 98$ pct density at $540 \mathrm{MPa}$. The $\mathrm{W}$ reinforcement inhibits the densification process, resulting in increased levels of residual porosity. The compaction behavior was evaluated using a modified Heckel equation, while the porosity dependence of the ultrasonically determined elastic modulus was described by a site percolation approach. Postcompaction sound-wave velocity and Vicker's hardness measurements show $<5$ pct anisotropy between the axial (pressing) and radial directions. The mechanical characterization illustrates the competing effects of the $\mathrm{W}$ reinforcement and the associated residual porosity.

DOI: $10.1007 / \mathrm{s} 11661-009-9935-9$

(C) The Author(s) 2009. This article is published with open access at Springerlink.com

\section{INTRODUCTION}

POWDER metallurgy is a useful route to the nearnet-shape fabrication of engineering components for a variety of applications. It allows consolidation without a melt-processing step, which can be advantageous for high-melting-point materials such as the refractory metals or ceramics. It is also useful for forming particulate composites from materials with greatly differing properties, such as oxide-dispersion-strengthened steels ${ }^{1 f}$ or, as in the present case, Bi-W materials for environmentally friendly ammunition. Roomtemperature powder compaction is an important part of this method, whether as a primary consolidation process or as a precursor to sintering. Models describing the behavior of powders during compaction are a useful tool for comparing different materials or preparation procedures (additives, milling, particle sizes, etc.) and for estimating porosity based on the compaction conditions. The various empirical models are probably the most widely applied method for the evaluation of compaction behavior, ${ }^{[2-5]}$ however, it can be difficult to

L. PETER MARTIN, Engineer, formerly with the Condensed Matter and Materials Science Division, Lawrence Livermore National Laboratory, is with Tyco Thermal Controls, Menlo Park, CA 94025. ANDREA M. HODGE, formerly Metallurgist, Condensed Matter and Materials Science Division, Lawrence Livermore National Laboratory, is Assistant Professor, Aerospace and Mechanical Engineering Department, University of Southern California, Los Angeles, CA 90089. GEOFFREY H. CAMPBELL, Scientific Capability Leader for Ultrafast Materials Science, is with the Condensed Matter and Materials Science Division, Lawrence Livermore National Laboratory, Livermore, CA 94550. Contact e-mail: ghcampbell@1lnl.gov

Manuscript submitted June 15, 2007.

Article published online July 30, 2009 directly correlate the fitting parameters with the actual physical characteristics of the materials. In addition, there exist relatively few studies comparing fitting parameters with the measured physical properties, ${ }^{[5,6]}$ particularly for composite mixtures. Similar comments can be made with respect to the analysis of the dependence of the mechanical properties on porosity, for which the most widely applied models are also largely empirical. ${ }^{[7]}$ In particular, the elastic properties are highly dependent upon the microstructure, which can be difficult to quantify in a way that is useful as input to theoretical models. Empirical modeling of both the compaction and mechanical properties can provide useful quantitative measures of the compaction behavior and the poroelastic response and can provide valuable insight into the processes involved.

In the present study, the compaction of composite mixtures of $\mathrm{Bi}$ and $\mathrm{W}$ powders is evaluated within the context of the modified Heckel equation. ${ }^{[5]}$ As expected, the powder mixtures behave similarly to the matrix (Bi) powder, but require higher applied pressure to reach comparable density due to a stress shielding effect of the reinforcing phase.$^{[8-13]}$ An experimental configuration is used in which compaction is performed incrementally, and the data are acquired in situ (without removing the compact from the die) but at zero applied pressure. Longitudinal sound-wave velocity measurements were also performed in situ, allowing calculation of the stiffness of the compacts in the pressing direction. The pore-free stiffness is estimated from the compaction data using a percolation model, and the compositional dependence compares favorably with the theoretical bounds proposed by Hashin and Shtrikman. ${ }^{[14]}$ Postcompaction density and sound-wave velocity measurements were also made ex situ (outside the die). 
The ex-situ measurements were used to evaluate the isotropy of the powder compacts and to calculate the bulk, shear, and Young's moduli. These moduli are modeled using the Mori-Tanaka (MT) effective field theory $^{[15]}$ to evaluate the effect of the $\mathrm{W}$ and porosity phases in the Bi matrix. Finally, Vicker's hardness measurements were performed to estimate the variation in strength with composition. The composition dependence of the measured hardness is shown to be qualitatively similar to that of the yield stress predicted by the Heckel analysis of the compaction data.

\section{EXPERIMENTAL}

The Bi-W powder metal composites were fabricated by cold compacting mixtures of commercial $\mathrm{Bi}$ and $\mathrm{W}$ powders (Alfa Aesar 10111 and 10400, respectively, Alfa Aesar, Ward Hill, MA). Typical bulk properties for Bi and $\mathrm{W}$ are given in Table I for reference. The powders were stored and handled under $\mathrm{N}_{2}$, with an $\mathrm{O}_{2}$ level $<200$ ppm. Figures 1(a) and (b) show scanning electron microscopy (SEM) micrographs of the loose powders. In Figure 1(a), the Bi powder can be seen to exhibit particle sizes ranging from $<1$ to $>30 \mu \mathrm{m}$, with a random shape characteristic of milling. In Figure 1(b), the $\mathrm{W}$ is comparatively finer, with a more uniform particle size. Figure 1(c) shows particle size analyses for the two powders. ${ }^{[16]}$ The particle size analyses indicate that the Bi powder has a $D_{50}=10 \mu \mathrm{m}$, while the $\mathrm{W}$ powder has a $D_{50}=5.8 \mu \mathrm{m}$. The analysis also shows the $\mathrm{W}$ to be somewhat bimodal, with a secondary peak at approximately $1.5 \mu \mathrm{m}$. An X-ray diffraction analysis was performed on the powders to evaluate the phase purity. The major $\mathrm{Bi}_{2} \mathrm{O}_{3}$ peak was barely resolved and no other oxides were detectable. This indicates that the oxide content is less than $\sim 5$ pct. The powders were mixed for 3600 seconds (1 hour) in a Nalgene jar (Thermo Scientific, Rochester, NY) using a Turbula mixer (Will A. Bachofen, Basel, Switzerland). Nylon spheres $12.5 \mathrm{~mm}$ in size were used as media to assist the process and could be separated using a coarse sieve. The mixed powders were then poured into a double-ended stainless steel cylindrical die (inner diameter $15.88 \mathrm{~mm}$ ) and leveled using a straight edge aligned with the top of the die. The loaded die was removed from the glovebox for compaction of the powders.

Uniaxial compaction was performed in air using a standard laboratory hydraulic press. The applied force was monitored using an Interface 1220-AF load cell

Table I. Bulk Properties of $\mathrm{Bi}$ and $\mathrm{W}$

\begin{tabular}{lcc}
\hline Property & W & Bi \\
\hline Density $\left(\mathrm{g} / \mathrm{cm}^{3}\right)$ & 19.3 & 9.8 \\
L-wave velocity $(\mathrm{mm} / \mu \mathrm{s})$ & 5.18 & 2.18 \\
$C_{33}(\mathrm{GPa})$ & 518 & 50 \\
S-wave velocity $(\mathrm{mm} / \mu \mathrm{s})$ & 2.87 & 1.10 \\
Bulk modulus $(\mathrm{GPa})$ & 310 & 35 \\
Shear modulus $(\mathrm{GPa})$ & 156 & 12 \\
Poisson's ratio & 0.29 & 0.35 \\
\hline
\end{tabular}

(Interface, Scottsdale, AZ) and 9840 gage. The compaction was performed in incremental steps from $\sim 1$ to $540 \mathrm{MPa}$. After each step, the pressure was relieved from the mold and the thickness of the powder compact was determined in situ (without removing from the
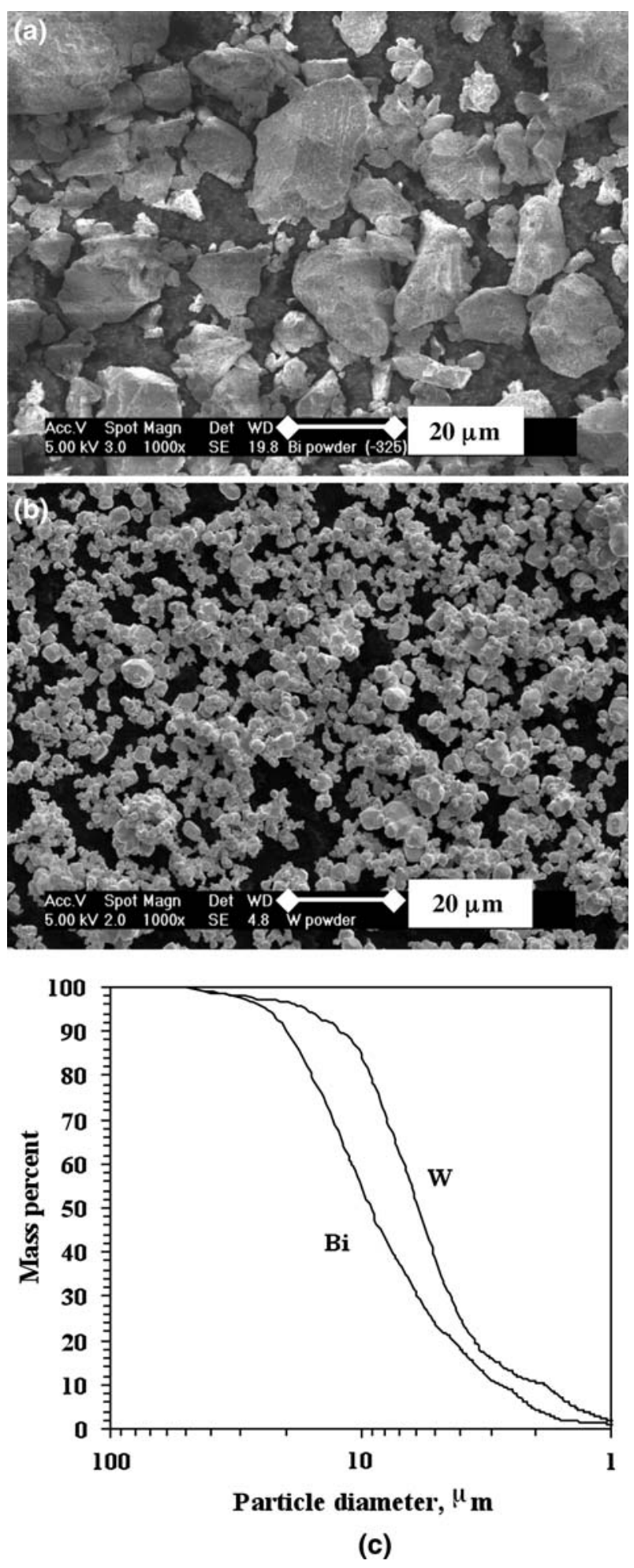

Fig. 1-(a) SEM micrograph of Bi powder (top), (b) SEM micrograph of $\mathrm{W}$ powder (bottom), and (c) particle size analysis of Bi and $\mathrm{W}$ powders. 
mold). This was done by measuring the distance between the opposing ends of the upper and lower punches and comparing that distance with the same dimension measured on the empty mold. The $5-\mathrm{MHz}$ longitudinal sound-wave velocity in the powder compact was also determined in situ at zero pressure. This was done by measuring the time of flight required for the sound waves to transit between the end faces of the two punches and comparing the measured time with the corresponding value for the empty mold. The velocity was calculated as the ratio of the thickness of the compact and the time required for the sound waves to travel through the compact. Given the known diameter of the mold and the mass of the compact measured at the end of the process, the apparent density $\rho$ after each compaction step could be calculated without removing the sample from the mold until the final compaction step was completed.

The apparent density $\rho$ was also determined ex situ (postcompaction) from the mass, diameter, and thickness of the compacted pellets measured outside the mold after the final compaction step. The thickness was determined by averaging multiple measurements made with a micrometer at the center and around the perimeter of each sample. The full (pore-free) density of the powder mixtures $\rho_{o}$ was calculated using a rule of mixtures:

$$
\rho_{o}=\sum_{n=1,2} f_{n} \rho_{n}
$$

where $f_{n}$ and $\rho_{n}$ are the volume fraction and density, respectively, of phase $n$. The apparent density $\rho$ is expressed as a fraction of the full density $\rho_{o}$ via the fractional density $\rho_{f}$ :

$$
\rho_{f}=\frac{\rho}{\rho_{o}}
$$

and the volume fraction of porosity $f_{p}$ is given by

$$
f_{p}=1-\rho_{f}=1-\frac{\rho}{\rho_{o}}
$$

The initial pore fraction $f_{p i}$ was determined from the density of the compact after the initial application of a slight pressure, $\sim 1 \mathrm{MPa}$.

Contact ultrasonic measurements were performed ex situ using 5-MHz longitudinal and shear-wave contact transducers made by the Panametrics (Olympus NDT, Waltham, MA) and recorded on an oscilloscope. The exsitu measurements were made with a gel couplant between the transducer and the part. The time of flight required for the sound waves to pass through the sample was determined by the pulse-echo overlap method, and the sound-wave velocity $V$ was determined from

$$
V=\frac{2 l}{t_{p e}}
$$

where $l$ is the thickness and $t_{p e}$ is the pulse-echo time of flight. The factor of 2 appears because, in this configuration, the measured time of flight is the time required for the sound waves to travel though the sample twice, i.e., from the transducer to the opposite surface and back. Vicker's hardness testing using a 100-g load was performed on polished surfaces normal to both the axial (plan view) and radial (cross-sectional view), per the directions in ASTM E 385-06.

\section{RESULTS AND DISCUSSION}

\section{A. Compaction Behavior}

The open symbols in Figure 2 show the fractional density $\rho_{f} v s$ the compaction pressure for the different volume fractions of $\mathrm{W}, f_{W}=0.0,0.073,0.23$, and 0.39 . The compaction was performed incrementally, as described earlier, with measurements performed at zero pressure between compaction increments. Thus, each series of points represents a single sample that was characterized in situ after each additional increment in pressure. Two sets of data are shown for each $f_{W}$. The data for $f_{W}=0$ represent the unreinforced $\mathrm{Bi}$ powder, which compacts to an apparent density of $\rho_{f} \sim 0.98$ at $540 \mathrm{MPa}$. At all compaction pressures, a higher $f_{W}$ results in a lower $\rho_{f}$, indicating that the $\mathrm{W}$ inclusions inhibit the densification process. Furthermore, at low compaction pressures, the effect of the $\mathrm{W}$ inclusions is more pronounced than at high pressures. For example, after compaction at $90 \mathrm{MPa}$, the fractional densities at $f_{W}=0.0$ and 0.39 are $\rho_{f} \sim 0.86$ and 0.70 , respectively. After compaction to $540 \mathrm{MPa}, \rho_{f} \sim 0.98$ and 0.89 , respectively, which is a significantly smaller differential. This behavior is consistent with published reports of the compaction of composite mixtures of $\mathrm{Bi}$ and $\mathrm{Ta}$ powders, $\mathrm{Pb}$ and steel spheres, $\mathrm{Pb}$ and alumina powders, and plasticene spheres and glass beads. ${ }^{[9,11,17]}$ In contrast, materials such as graphite-reinforced Fe; SiC-,

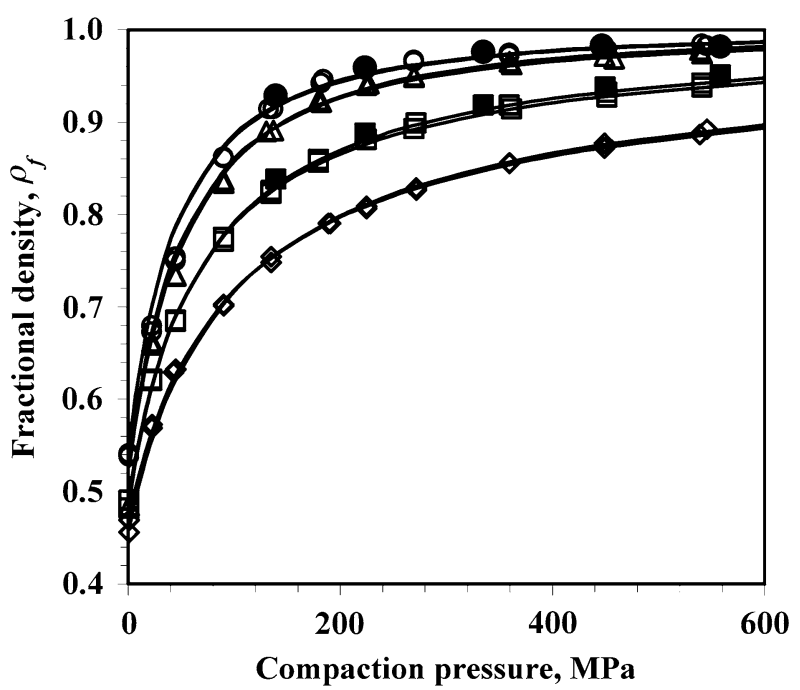

Fig. 2-Fractional density $v s$ compaction pressures for $f_{W}=0.00$ $(\bigcirc, \bullet), 0.073(\Delta), 0.23(\square, \boldsymbol{\square})$, and $0.39(\diamond)$. Open symbols represent data from the incremental press-and-measure-technique described in the text. Filled symbols represent data from samples pressed directly to the indicated compaction pressures and characterized ex situ after pressing. Lines represent the best fit of Eq. [8] to the incremental compaction data. 
steel-, and zirconia-reinforced $\mathrm{Al}$; and W-reinforced $\mathrm{Cu}$ exhibit an opposite effect in which inhibited densification becomes more pronounced at higher compaction pressures. ${ }^{[2-5,8,10,12,13,18]}$ The difference in behavior for these systems may relate to the ductility of the matrix material $(\mathrm{Pb}, \mathrm{Bi}$, or plasticene vs $\mathrm{Al}, \mathrm{Fe}$, or $\mathrm{Cu})$.

Limited compaction data are also shown by the solid symbols in Figure 2 for two series of samples $\left(f_{W}=0.0\right.$ and 0.23 ) pressed directly to the indicated compaction pressures and characterized ex situ after pressing. This process is distinct from the incremental press-andmeasure process, in that each series of data represents several powder compacts, each of which was taken directly to the indicated pressure in a single step. The data for the two compaction methods compare favorably, confirming that the incremental pressure increaserelease cycle does not yield significantly different compaction behavior from single-step compaction.

A modified Heckel equation ${ }^{[2,5]}$ was fit to the compaction data in Figure 2 in order to quantify the relative behavior at different $f_{W}$. The Heckel equation was first published by Shapiro and later by Konopicky, ${ }^{[19,20]}$ and relates the compaction process to a reduction in porosity that behaves as a first-order reaction:

$$
\frac{-d f_{p}}{d P}=K f_{p}
$$

where $P$ is the compaction pressure and $K$ is a constant analogous to a reaction rate. Later, Heckel investigated a number of metal powders and identified an empirical relationship between $K$ and $\sigma_{o}$, the yield stress, in the absence of porosity: ${ }^{[2,4,5,21]}$

$$
K=\frac{1}{3 \sigma_{o}}
$$

The term $3 \sigma_{o}$ is known as the yield pressure (sometimes as the Heckel yield pressure). An empirical correlation has been made between $3 \sigma_{o}$ and the measured hardness and Young's modulus for a wide range of materials. ${ }^{[6]}$ The classic Heckel equation is derived by substituting Eq. [6] into Eq. [5] and integrating. The resultant relation predicts a linear dependence of $\ln (1 /$ $f_{p}$ ) on the compaction pressure $P$ and has been widely applied to a variety of materials. However, it has been observed that a pronounced curvature often occurs outside the central portion of the compaction diagram. ${ }^{[2,5]}$ Several approaches have been taken toward modification of the Heckel equation, to improve the capability to fit a broader range of experimental data. One of the more effective approaches has been to address the pressure dependence of $\sigma_{o}{ }^{[5,22]}$ The pressure dependence of $\sigma_{o}$ arises from the increasing constraint caused by neighboring particles as the compaction decreases, $f_{p}$. This can be done in a variety of ways, one of which is to replace $\sigma_{o}$ in Eq. [6] by ${ }^{[5]}$

$$
\sigma=\sigma_{o}+k_{1} P
$$

where $\sigma$ is the pressure-dependent yield stress and $k_{1}$ is a function of the Poisson's ratio $v$. Substituting Eq. [6] and Eq. [7] into Eq. [5] and integrating yields the modified Heckel equation:

$$
\ln \left(\frac{1}{f_{p}}\right)=\ln \left(\frac{1}{f_{p o}}\right)+\frac{1}{3 k_{1}} \ln \left[1+\frac{k_{1} P}{\sigma_{o}}\right]
$$

where $f_{p o}$ represents some initial-state pore fraction. In the original derivation, the term $f_{p o}$ was postulated to represent the pour density of the powder. Later, the term $\ln \left(1 / f_{p o}\right)$ was redefined to be the sum of a term relating to the pour density and an offset term relating to the additional compaction associated with particle rearrangement. ${ }^{[2]}$ In that case, the pressure-dependent part of the Heckel relation is only intended to be valid at pore fractions below which particle rearrangement does not occur to any significant extent, and where the compact behaves as a coherent body capable of supporting enough of the applied stress to cause deformation of the particles.

Particle rearrangement (sliding, restacking, etc.) is generally accepted to be the primary densification mechanism during the first stage of compaction, in which significant densification occurs with negligible applied pressure. ${ }^{[2,5,19]}$ At the completion of the first stage of compaction, no further free movement of the particles is possible and the powder compact now begins to support (i.e., resist) the applied stress. Additional compaction occurs with the development of significant stress in the compact and proceeds via plastic deformation, or brittle fracture, of the particles. ${ }^{[5,23]}$ It is this latter process that is described by the Heckel equation, particularly for the case of plastic, rather than brittle, materials. The current method of determining $f_{p i}$ from the density of the compact after application of a light pressure is consistent with the idea that $f_{p o}$ corresponds to the pore fraction reached at the minimum pressure required to form a coherent compact. ${ }^{[21]}$ Thus, in this investigation, $f_{p o}$ was taken to be equal to the $f_{p i}$ described earlier. It should be noted that, due to the effects of particle size and shape distributions, a sharp transition from particle motion to plastic deformation is not expected; ${ }^{[23]}$ therefore, the pressure at which $f_{p i}$ was determined $(\sim 1 \mathrm{MPa})$ is somewhat arbitrary. However, it provides a repeatable initial value that should be more relevant to the process than ex-situ measurements such as the pour or tap density.

Eq. [8] was fit to the incremental compaction data from Figure 2 using a least squares regression analysis to determine optimal values for $k_{1}$ and $\sigma_{o}$. The input values for $f_{p i}$ were determined experimentally, as described earlier. The results are plotted as the solid lines in Figure 2. Clearly, Eq. [8] accurately describes the compaction behavior over the full range of pressures and compositions. The fitting parameters and $f_{p i}$ used to generate the curves are given in Table II. There, $\sigma_{o}$ is reported to be $\sim 15.1 \mathrm{MPa}$ for $f_{W}=0$, and increases with increasing $f_{W \cdot}$ A similar effect was reported for Ta-reinforced $\mathrm{Bi},{ }^{[17]}$ where the compaction was performed over the range 140 to $550 \mathrm{MPa}$ in the single-step process used to produce the data for the filled symbols in Figure 2. There does not appear to be any significant published work evaluating the correlation between the yield strength determined from mechanical testing and the yield parameters determined from Eq. [8]. However, the calculated $\sigma_{o}$ values compare favorably with the 
Table II. Fitting Parameters $k_{1}$ and $\sigma_{o}$ Used to Fit Equation [8] to Compaction Data from Figure 2; Also Shown Are Experimentally Determined $f_{p i}$ and Poisson's Ratio (v) Predicted by Equation [9]

\begin{tabular}{lcccc}
\hline$f_{W}$ & $f_{p i}{ }^{*}$ & $k_{1}$ & $\sigma_{o}$ & $v$ \\
\hline 0.00 & 0.459 & 0.211 & 15.3 & 0.276 \\
0.00 & 0.462 & 0.207 & 14.9 & 0.274 \\
0.073 & 0.481 & 0.211 & 18.2 & 0.291 \\
0.073 & 0.481 & 0.242 & 16.7 & 0.293 \\
0.23 & 0.518 & 0.360 & 21.9 & 0.378 \\
0.23 & 0.509 & 0.329 & 23.3 & 0.363 \\
0.39 & 0.544 & 0.450 & 31.9 & 0.375 \\
0.39 & 0.531 & 0.469 & 32.8 & 0.381 \\
$0.00^{\mathrm{a}}$ & 0.660 & 0.233 & 8.66 & 0.288 \\
$0.00^{\mathrm{b}}$ & 0.460 & 0.218 & 12.8 & 0.280 \\
$0.00^{\mathrm{c}}$ & 0.660 & 0.246 & 9.22 & 0.294 \\
$0.00^{\mathrm{c}}$ & 0.660 & 0.240 & 9.24 & 0.292 \\
\hline
\end{tabular}

*Experimentally determined.

${ }^{a}$ From Ref. 16, using experimentally determined $f_{p i}$.

${ }^{\mathrm{b}}$ From Ref. 16, using $f_{p i}=$ pour density.

${ }^{\mathrm{c} C}$ Current data, using $f_{p i}=$ pour density.

yield strengths determined from the compression testing of polycrystalline Bi at different temperatures and strain rates. ${ }^{[24]}$ In Reference 24, room-temperature yield occurred between $\sim 13$ and $24 \mathrm{MPa}$, depending on the strain rate $\left(2 \times 10^{-4}\right.$ to $2 \times 10^{-2}$, respectively). Thus, the calculated $\sigma_{o}$ for Bi appears to be in an appropriate range to provide a representative estimate of the yield strength.

In Reference 17, Eq. [8] was fit to the limited singlestep compaction data (filled symbols) for $f_{W}=0$ (pure Bi) shown in Figure 2. In that case, however, the $f_{p i}$ used in the current study was not experimentally accessible, and a value based on the pour density of the powder was used, $f_{p i}^{\text {pour }}=0.66$, which was considerably higher than the $f_{p i} \sim 0.46$ measured by the current technique. The result of that analysis, as well as a second analysis of the current data using $f_{p i}=f_{p i}^{\text {pour }}=0.66$, are shown at the bottom of Table II. For the current data, shifting $f_{p i}$ from $\sim 0.46$ to 0.66 reduces the calculated $\sigma_{o}$ from $\sim 15.1$ to $\sim 9.2 \mathrm{MPa}$, which is comparable to that from the earlier work, 8.66 MPa. Conversely, applying the current experimentally determined $f_{p i} \sim 0.46$ to the data from Reference 17 yields $\sigma_{o}=12.8 \mathrm{MPa}$. Thus, most of the discrepancy between the $\sigma_{o}$ values calculated here and those in Reference 17 results from the selection of the value for $f_{p i}$. In addition, there is probably some refinement of the calculation gained by the broader range of pressures used for the current investigation, in particular, the pressures $<100 \mathrm{MPa}$ in which $\rho_{f}$ changes rapidly with pressure, which provides a more complete "view" of the compaction process. This analysis highlights the importance of properly identifying the initial state of the powder compact before applying this type of model to the compaction process.

It has been proposed that the values for $k_{1}$ listed in Table II can be correlated with the Poisson's ratio (v) via ${ }^{[5]}$

$$
k_{1}=\frac{2 v^{2}}{(1-v)}
$$

The values for $v$ calculated using Eq. [9] are listed in Table II for the different $f_{W}$. The range of values $(0.279$ to 0.380 ) is reasonable for metallic materials and is qualitatively consistent with the behavior observed in Reference 17. However, the observed trend of increasing $v$ with increasing $f_{W}$ is not consistent with the accepted values of $v$ for $\mathrm{Bi}$ and $\mathrm{W}$ reported in Table I: 0.346 and 0.285 , respectively. The behavior appears to indicate that Eq. [9] is not valid for these materials, probably because it neglects the effects of elastic strain in the die, the pressure dependence of $v$, and any potential anisotropy in the powder compact.

Figure 3(a) shows the longitudinal wave velocity, designated as $V_{3}$, measured in situ along the compaction direction $v s$ the volume fraction of porosity $f_{p}$ determined via Eq. [3]. The data show a monotonic increase in $V_{3}$ with decreasing $f_{p}$ for all compositions. Further, at constant $f_{p}$, the data show increasing $V_{3}$ with increasing $f_{W}$. This behavior results from the competing effects of the increased stiffness associated with the $\mathrm{W}$ reinforcement, and from the reduced stiffness caused by the resultant residual porosity. Ultrasonic sound-wave velocity has been widely applied to the characterization of porous powder compacts, ${ }^{[25-28]}$ because it is intrinsically related to the elastic moduli (stiffness) and density by the well-known relation

$$
C=V^{2} \rho
$$

where $C$ is the elastic stiffness and $V$ is the velocity of sound. The particular modulus described by Eq. [10] depends on the mode and, for anisotropic materials, the direction of the propagation of the sound waves. If we designate a coordinate system for our cylindrical samples in which the $x_{3}$ axis is in the axial (pressing) direction and the two orthogonal directions, $x_{1}$ and $x_{2}$, are in the radial (transverse) plane, then Eq. [10] becomes

$$
C_{33}=V_{3}^{2} \rho
$$

In Eq. [11], $C_{33}$ represents the principal axial component of the elastic stiffness tensor $C_{i j}$, which relates stress and strain through Hooke's law:

$$
\left[\begin{array}{l}
\sigma_{11} \\
\sigma_{22} \\
\sigma_{33} \\
\tau_{23} \\
\tau_{31} \\
\tau_{12}
\end{array}\right]=\left[\begin{array}{cccccc}
C_{11} & C_{12} & C_{13} & 0 & 0 & 0 \\
C_{12} & C_{11} & C_{13} & 0 & 0 & 0 \\
C_{13} & C_{13} & C_{33} & 0 & 0 & 0 \\
0 & 0 & 0 & C_{44} & 0 & 0 \\
0 & 0 & 0 & 0 & C_{44} & 0 \\
0 & 0 & 0 & 0 & 0 & C_{66}
\end{array}\right]\left[\begin{array}{l}
\varepsilon_{11} \\
\varepsilon_{22} \\
\varepsilon_{33} \\
\gamma_{23} \\
\gamma_{31} \\
\gamma_{12}
\end{array}\right]
$$

where $C_{66}=1 / 2\left(C_{11}-C_{12}\right)$ and $\sigma, \tau, \varepsilon$, and $\gamma$ represent the normal and shear stresses and the normal and shear strains, respectively. The $C_{i j}$ matrix in Eq. [12] is expressed for the case of transverse isotropy in order to reflect the symmetry of the uniaxial 


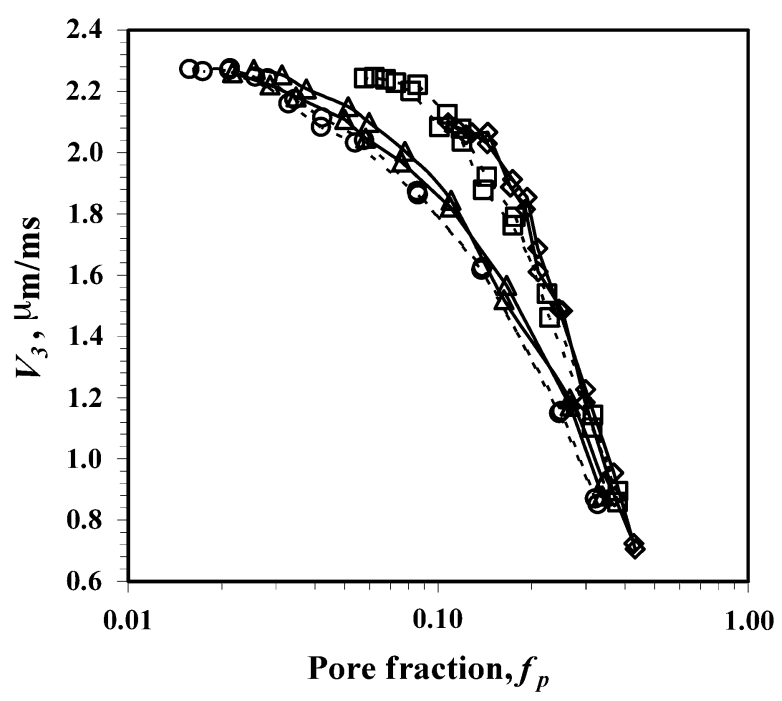

(a)

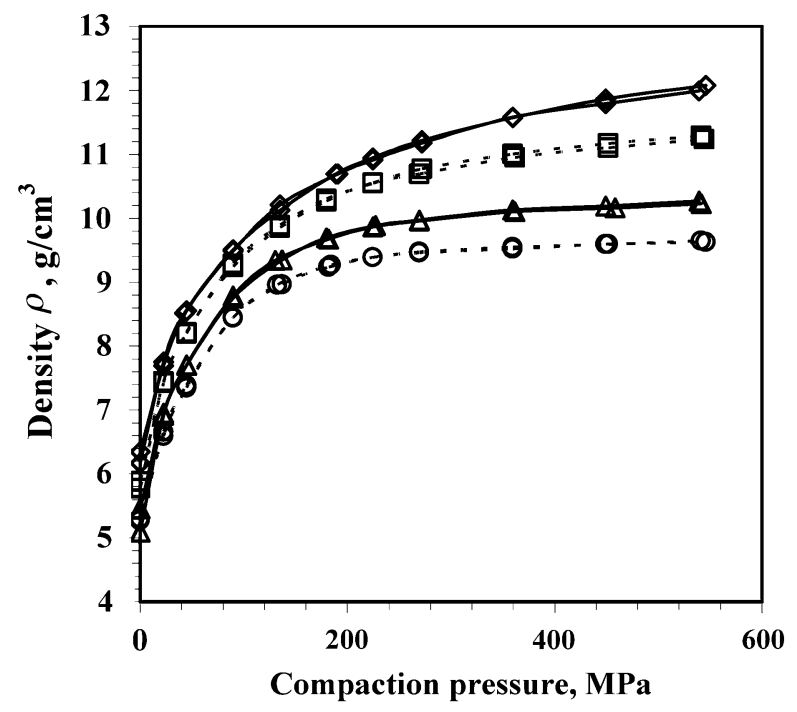

(b)

Fig. 3-(a) Longitudinal sound-wave velocity measured along the axial (pressing) direction $\left(V_{3}\right)$ and $(b)$ apparent density $(\rho)$, both measured in situ at zero pressure. Data are shown for $f_{W}=0.00$ $(\bigcirc), 0.073(\Delta), 0.23(\square)$, and $0.39(\diamond)$.

pressing configuration. For complete isotropy, Eq. [12] simplifies significantly due to the following relations:

$$
\begin{aligned}
& C_{11}=C_{33} \\
& C_{44}=C_{66} \\
& C_{12}=C_{13}=\left(C_{11}-2 C_{44}\right)
\end{aligned}
$$

In either case, Eq. [11] takes into account the isotropy of the material, and the velocity data in Figure 3(a) can be used to calculate $C_{33}$ via Eq. [11] and the apparent density shown in Figure 3(b).

The data in Figure 3(b) correspond to the $\rho_{f}$ data shown in Figure 2, but the apparent densities are expressed in grams per cubic centimeter. It is not surprising that a comparison of Figures 2 and 3(b) show that, while $\rho_{f}$ decreases with increasing $f_{W}$ due to the

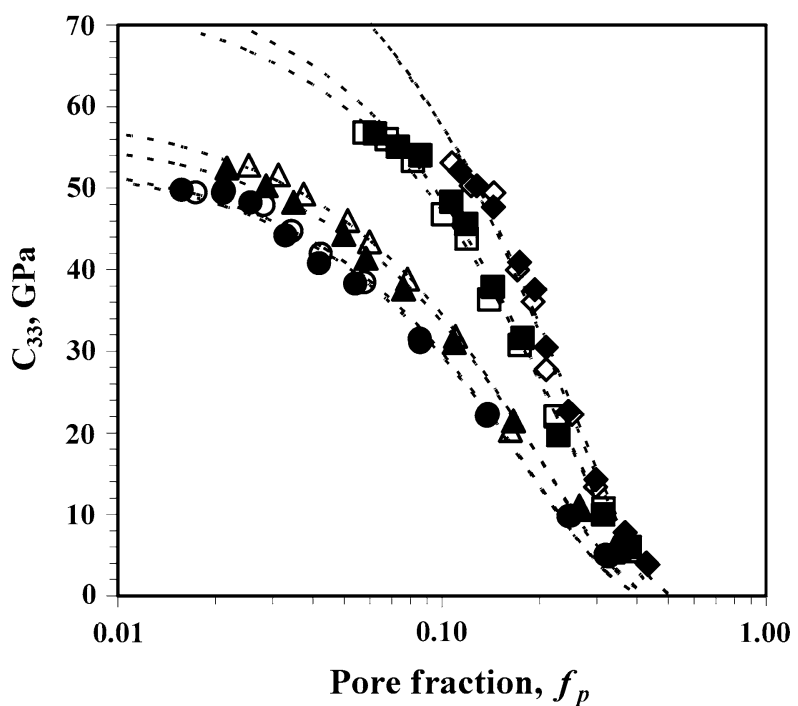

Fig. 4-The $C_{33}$, calculated from Eq. [11] using data in Figs. 3(a) and (b), vs fraction of porosity $f_{p}$. The $C_{33}$ are shown for $f_{W}=0.00$ $(\bigcirc, \bullet), 0.073(\Delta, \mathbf{\Lambda}), 0.23(\square, \mathbf{\square})$, and $0.39(\diamond, \diamond)$. Dotted lines indicate the best fit of Eq. [16] to each data set (two sets at each $f_{W}$ ).

associated increase in $f_{p}$, the actual apparent density $\rho$ increases due to the significantly higher density of $\mathrm{W}$ relative to $\mathrm{Bi}$ (Table I). Figure 4 shows the calculated $C_{33} v s f_{p}$ for the different $f_{W}$ values. The trends in the $C_{33}$ data are qualitatively similar to those observed in the velocity data shown in Figure 3(a), except that the relative contribution of the apparent density $\rho$ causes the effect of $f_{W}$ to be more apparent. That is, the data at different $f_{W}$ are somewhat more "spread out" in Figure 4 than in Figure 3(b). Physical modeling of the elastic behavior requires detailed knowledge of the microstructure (Bi, W, and porosity), which is difficult to obtain and apply quantitatively. However, numerous empirical and semiempirical models exist that have been proposed to account for the effects of a second phase (generally porosity) on the elastic moduli. ${ }^{[2-32]}$ These models often apply assumptions that may not be realistic; they can result in fitting parameters that are difficult to relate to the actual material properties. Nevertheless, when properly applied, they can provide quantitative comparisons between different materials or processes, and can yield valuable insight into the observed behavior.

One approach to modeling the elastic behavior has been to apply the percolation theory to predict the effect of porosity. ${ }^{[23,33]}$ The percolation theory has been applied to a broad range of physical phenomena, including the gelation of polymers, the spreading of fluid in porous systems, the design of pharmaceutical tablets, and the compositional dependence of the hardness and elastic moduli in binary and ternary powder compacts. ${ }^{[23,34]}$ Random percolation theory is a statistical approach in which the system is described by an underlying lattice, each point of which is randomly occupied by one of the system components. A cluster is defined as a group of neighboring sites occupied by the same component (i.e., "A" in a binary system composed 
of phases A and B). The point at which a cluster just percolates a system is known as the percolation threshold. The theory relates the apparent value of a property $X$ to the site occupation (or bond) probability $\Phi$ by

$$
X=X_{o} \Phi^{b}
$$

where $X_{o}$ is the value of the property for the matrix phase alone and $b$ is the critical exponent describing the change of $X$ with $\Phi .^{[33]}$ Theoretically, the value of $b$ is dependent solely on the fractal dimension of the process, with a three-dimensional structure of round spheres yielding $b \sim 2.1 .^{[32,33,35]}$ However, it has been reported that there can be significant experimental deviations from this predicted value, thus giving a "characteristic" rather than a "critical" exponent. ${ }^{[35-37]}$ These deviations may result from any of the following: the finite size of the system (the theory treats an infinitely large system $^{[38]}$, application of an inappropriate lattice model (discrete lattice, continuum, etc.), application of an inappropriate percolation type (random site, random bond, etc.), anisomorphous particle shape, or anisotropy in the powder compact. ${ }^{[35]}$

The compaction of powders, and the concurrent reduction in porosity, can be treated as a site percolation phenomenon. ${ }^{[23]}$ The probability that a site is occupied can then be related to the fractional density by

$$
\Phi=\frac{\rho_{f}-\rho_{c r}}{1-\rho_{c r}}
$$

where $\rho_{c r}$ is the percolation threshold. This has been associated with the tap density for a variety of powders, ${ }^{[23,39]}$ but is most appropriately described as the fractional density at which (during compaction) particle rearrangement ceases and further densification occurs by the buildup of a significant compressional force. ${ }^{[23,38]}$ Treating the powder compact as a binary system consisting of porosity and an apparent matrix of the $\mathrm{Bi}$ and $\mathrm{W}$ powder and, recalling Eq. [3], Eq. [14], and Eq. [15], then yield the following relation:

$$
X=X_{o}\left(\frac{f_{p, c r}-f_{p}}{f_{p, c r}}\right)^{b}
$$

where $f_{p, c r}$ is taken to be the experimentally determined $f_{p i}$ discussed previously. Van Veen et al. followed a similar approach to estimate the pore-free elastic moduli in pressed compacts of $\mathrm{NaCl}$ and starch. ${ }^{[35]}$ Taking $X$ and $X_{o}$ to be $C_{33}$ and $C_{33, o}$, respectively, Eq. [16] was fit to the data shown in Figure 4, in which the dotted lines show the best fit for each $f_{W}$. The fitting parameters used in Eq. [16], $C_{33, o}$ and $b$, are shown in Table III for each of the compositions. The calculated values for $b$ fall close to the theoretically predicted value of 2.1 and appear to exhibit a decreasing trend with increasing $f_{W}$. The origin of this trend is not clear at present, but may be due to the asphericity of the initial powders. In contrast, the predicted $C_{33, o}$ values show an increase with $f_{W}$, which is an expected consequence of the increased stiffness associated with the $\mathrm{W}$ reinforcement.

The values of $C_{33, o}$ are shown $v s f_{W}$ in Figure 5. Also shown are the theoretical bounds for a two-phase
Table III. Fitting Parameters $C_{33, o}$ and $b$ Used to Fit Equation [16] to $C_{33}$ Data from Fig. 4; Also Shown Are Experimentally Determined $f_{p i}$

\begin{tabular}{lccc}
\hline$f_{W}$ & $f_{p i}{ }^{*}$ & $C_{33, o}$ & $b$ \\
\hline 0.00 & 0.459 & 54.1 & 2.41 \\
0.00 & 0.462 & 53.7 & 2.45 \\
0.073 & 0.481 & 56.9 & 2.26 \\
0.073 & 0.481 & 59.6 & 2.35 \\
0.23 & 0.518 & 77.4 & 2.16 \\
0.23 & 0.509 & 74.3 & 2.07 \\
0.39 & 0.544 & 91.6 & 2.27 \\
0.39 & 0.531 & 89.9 & 2.09 \\
\hline
\end{tabular}

*Experimentally determined.

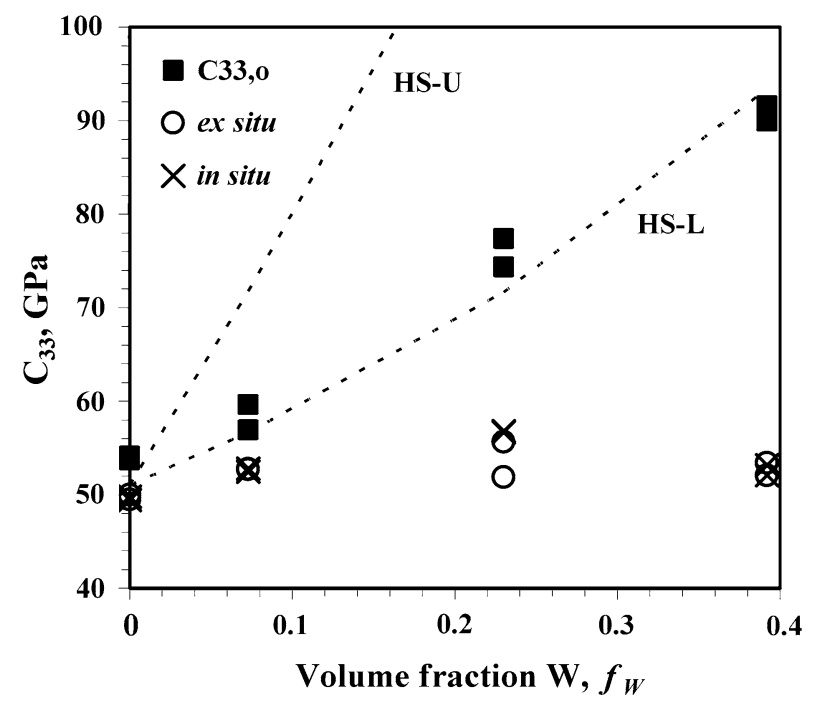

Fig. 5-Predicted pore-free modulus, $C_{33, o}(\mathbf{\square})$, determined from fitting Eq. [16] to the data in Fig. 4, vs $f_{W}$. Dotted lines represent the upper (HS-U) and lower (HS-L) Hashin-Shtrikman bounds calculated from Eqs. [17] through [21]. Also shown are the experimentally determined $C_{33}$ measured in situ $(\mathrm{X})$ and ex situ $(\mathrm{O})$.

medium proposed by Hashin and Shtrikman. ${ }^{[14]}$ The upper (HS-U) and lower (HS-L) Hashin-Shtrikman bounds are calculated from

$$
\begin{gathered}
B_{L}=B_{\mathrm{Bi}}+\frac{f_{\mathrm{W}}}{\frac{1}{B_{\mathrm{W}}-B_{\mathrm{Bi}}}+\frac{3 f_{\mathrm{Bi}}}{3 B_{\mathrm{Bi}}+4 G_{\mathrm{Bi}}}} \\
B_{U}=B_{\mathrm{W}}+\frac{f_{\mathrm{Bi}}}{\frac{1}{B_{\mathrm{Bi}}-B_{\mathrm{W}}}+\frac{3 f_{\mathrm{W}}}{3 B_{\mathrm{W}}+4 G_{\mathrm{W}}}} \\
G_{L}=G_{\mathrm{Bi}}+\frac{f_{\mathrm{W}}}{\frac{1}{G_{\mathrm{W}}-G_{\mathrm{Bi}}}+\frac{6 f_{\mathrm{Bi}}\left(B_{\mathrm{Bi}}+2 G_{\mathrm{Bi}}\right.}{5 G_{\mathrm{Bi}}\left(3 B_{\mathrm{Bi}}+4 G_{\mathrm{Bi}}\right)}} \\
G_{U}=G_{2}+\frac{f_{\mathrm{Bi}}}{\frac{1}{G_{\mathrm{Bi}}-G_{\mathrm{W}}}+\frac{6 f_{\mathrm{W}}\left(B_{\mathrm{W}}+2 G_{\mathrm{W}}\right)}{5 G_{\mathrm{W}}\left(3 B_{\mathrm{W}}+4 G_{\mathrm{W}}\right)}}
\end{gathered}
$$

where $B$ and $G$ are the bulk and shear moduli, respectively, and the subscripts $U$ and $L$ refer to the upper 
and lower bound, respectively. The $B$ and $G$ for the Bi and $\mathrm{W}$ were taken from Table I. The upper- and lower-bound bulk and shear moduli are, in turn, related to $C_{33}$ by

$$
C_{33}=\frac{4}{3} G+B
$$

The predicted $C_{33, o}$ values fall very close to the lower (HS-L) bound. As a point of interest, it has been shown that for $B$, the lower HS bound is an exact solution for the case of hard spherical particles in a soft matrix. ${ }^{[40]}$ The analysis implies that the predicted values of $C_{33, o}$ fall within an appropriate range to provide an estimate of the pore-free modulus for $\mathrm{Bi}-\mathrm{W}$ composites prepared by the present methodology. It should be noted that calculation of the bounds assumes the homogeneous mixing of the inclusion phase, as well as overall isotropy in the composite. These issues will be discussed in more detail later in this article.

\section{B. Postcompaction Characterization}

The density and axial sound-wave velocity $V_{3}$ were also measured ex situ after compaction to the maximum pressure of $540 \mathrm{MPa}$. The ex-situ values of $V_{3}$ generally agree with the in-situ measurements to $\leq 0.5 \mathrm{pct}$. The ex-situ $V_{3}$ and $\rho$ were used to calculate $C_{33}$ via Eq. [11] and the results are plotted $v s f_{W}$ in Figure 5 as $C_{33 \text {,ex }}$. The ex-situ values compare favorably with those measured in situ, as shown in the figure, agreeing in most cases to $\leq 1.0$ pct. As expected, as $f_{W}$ increases, the measured $C_{33}$ falls increasingly below the predicted value of $C_{33, o}$ due to the increase in $f_{p}$ (Figure 2). Optical microscopy, measurements of the sound-wave velocity vs direction, and Vicker's hardness testing were performed in order to facilitate further discussion of the mechanical properties and to quantify the anisotropy in the compacts.

Figure 6 shows optical micrographs for $f_{W}=0$, $0.073,0.23$, and 0.39 after compaction to $540 \mathrm{MPa}$. Two sets of images are shown, corresponding to two different directions within the microstructure. The "axial view" looks along the $x_{3}$ direction, and thus the pressing direction is into the plane of the image. The "radial view" looks along the $x_{1}-x_{2}$ plane, and thus the pressing direction is top to bottom in the image. At $f_{W}=0$, the images show a dense microstructure with a broad particle size distribution consistent with the particle size analysis from Figure 1. There is some visible heterogeneity in the microstructure, in that the average grain size in the axial image appears larger than in the radial image. Thus, when the microstructure is viewed along the pressing direction away from the die walls, the grains appear larger than when viewed normal to the pressing direction. The implication is that the microstructure is compressed more in the axial direction than in the radial direction, with the caveat that angularity and possible asymmetry of the Bi particles could influence particle settling and arrangement. This trend persists throughout the range of $f_{W}$ and may lead to some anisotropy in the mechanical properties. At $f_{W}=0.073$, the microstructure still appears dense, with the $\mathrm{W}$ reinforcement clearly visible as the slightly darker phase residing on the $\mathrm{Bi}$ particle boundaries. There is some evidence of small, isolated pores, particularly in areas where the $\mathrm{W}$ has agglomerated. At $f_{W}=0.23$, the residual porosity is clearly evident (it appears black in the image), and the network of $\mathrm{W}$ particles on the $\mathrm{Bi}$ particle boundaries has become more developed. It appears that the "width" of the W phase between the Bi particles has increased relative to that at $f_{W}=0.073$, and the $\mathrm{W}$ phase appears to be near percolation. At $f_{W}=0.39$, the size of the $\mathrm{W}$ regions has increased dramatically, leading to significantly more entrained porosity. In addition, the $\mathrm{W}$ is clearly percolated, particularly in the radial image. As this $\mathrm{W}$ network develops, it becomes capable of supporting more of the applied stress and, therefore, less of the stress is transferred to the $\mathrm{Bi}$ matrix.

That the microstructure appears slightly different when viewed from the axial and radial directions raises the possibility that the mechanical properties in the compacts may be anisotropic. It is well known that uniaxial pressing can lead to significant stress gradients during compaction. It is also known, however, that, as the compaction pressure becomes much greater than the yield stress of the powder, the radial pressure approaches the axial pressure. ${ }^{[5]}$ In order to quantify the state of isotropy in the compacts, the longitudinal and shear sound-wave velocities were measured as a function of the direction. Recalling the coordinate system in which the $x_{3}$ axis is in the axial (pressing) direction and the two orthogonal directions, $x_{1}$ and $x_{2}$, are in the radial (transverse) plane, there are five principal (i.e., on-axis) sound-wave velocities that can be measured: longitudinal wave propagating in the axial and radial directions $\left(V_{3}\right.$ and $\left.V_{1}\right)$, shear wave propagating in the axial direction $\left(V_{31}\right)$, shear wave propagating in the radial direction and polarized in the $x_{1}-x_{2}$ plane $\left(V_{12}\right)$, and shear wave propagating in the radial direction and polarized in the $x_{3}$ direction $\left(V_{13}\right)$. The first subscript indicates the propagation direction and the second subscript indicates the polarization direction (where applicable) for the shear waves. In a transversely isotropic solid, all of the nonzero stiffness components except $C_{13}$ can be determined from four of the velocities by ${ }^{[41,42]}$

$$
\begin{gathered}
C_{11}=\rho V_{1}^{2}, \quad C_{33}=\rho V_{3}^{2} \\
C_{44}^{\text {axial }}=\rho V_{31}^{2} \\
C_{66}=\frac{1}{2}\left(C_{11}-C_{12}\right)=\rho V_{12}^{2}
\end{gathered}
$$

The determination of $C_{13}$ requires off-axis measurements and is not relevant to the current discussion. From Eq. [13] and Eq. [22], it is also apparent that, in the case of isotropy, all components of the $C_{i j}$ matrix can be determined from just two sound-wave velocities, longitudinal $\left(V_{3}\right)$ and shear $\left(V_{31}\right)$. 

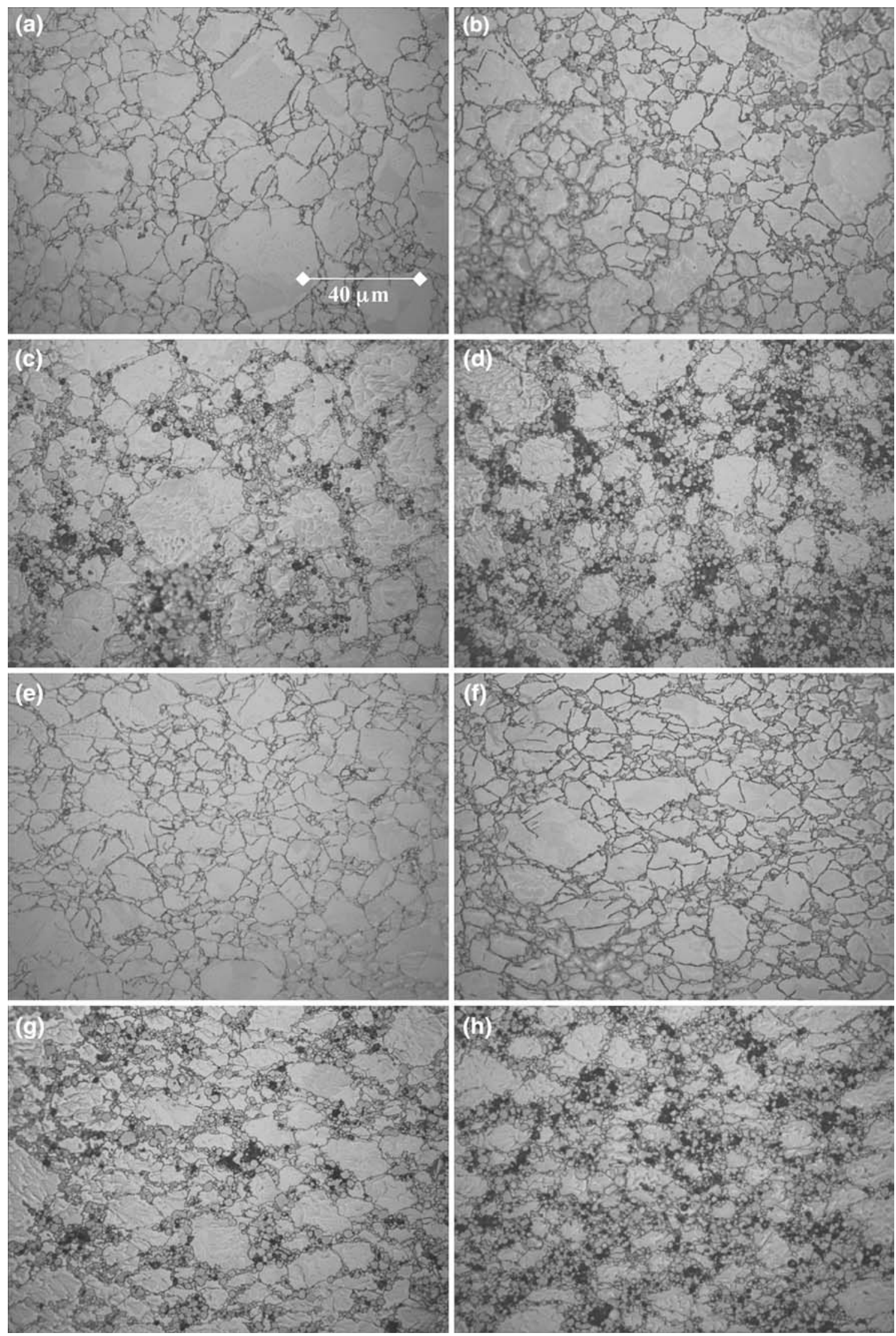

Fig. 6 - Optical images of composite microstructures looking along both axial and radial directions. For axial view $(a)$ through $(d)$, the pressing direction is into the page. For radial view (e) through $(h)$, the pressing direction is top to bottom. Images are for (a) and (e) $f_{W}=0.00$, (b) and (f) 0.073 , (c) and (g) 0.23 , and (d) and (h) 0.39 . Scale bar shown in the first image is valid for all images. 
A comparison of Eq. [12], Eq. [13], and Eq. [22] implies certain relations that may be used to quantify the isotropy of the material, ${ }^{[41]}$ namely:

$$
\begin{gathered}
\frac{C_{33}}{C_{11}}=\left(\frac{V_{3}}{V_{1}}\right)^{2} \\
\frac{2 C_{44}}{C_{11}-C_{12}}=\left(\frac{V_{31}}{V_{12}}\right)^{2}
\end{gathered}
$$

Eq. [23] and Eq. [24] provide a measure of the longitudinal (compressive) and shear anisotropy, with $C_{33}=C_{11}$ and $2 C_{44}=\left(C_{11}-C_{12}\right)$ indicating isotropy. These parameters are plotted $v s f_{W}$ in Figure 7, in which values of "1.00" correspond to complete isotropy. The deviation from isotropy is $<5$ pct for both parameters. The implication is that, for this material system, the assumption of isotropy will introduce an error of $\leq 5 \mathrm{pct}$ when relating the sound speeds to the elastic moduli. This assumption of isotropy is convenient for further analysis of the engineering elastic moduli within the context of the MT effective field model.

The so-called engineering elastic moduli can be related to the longitudinal and shear-wave velocity in isotropic materials by the following well-known relations:

$$
\begin{gathered}
G=\rho V_{S}^{2} \\
E=\rho V_{S}^{2} \frac{3 V_{L}^{2}-4 V_{S}^{2}}{V_{L}^{2}-V_{S}^{2}} \\
B=\rho\left(V_{L}^{2}-\frac{4}{3} V_{S}^{2}\right)
\end{gathered}
$$

where $E$ is the Young's modulus and $V_{L}$ and $V_{S}$ are the longitudinal $\left(V_{1}=V_{2}=V_{3}\right)$ and shear-wave

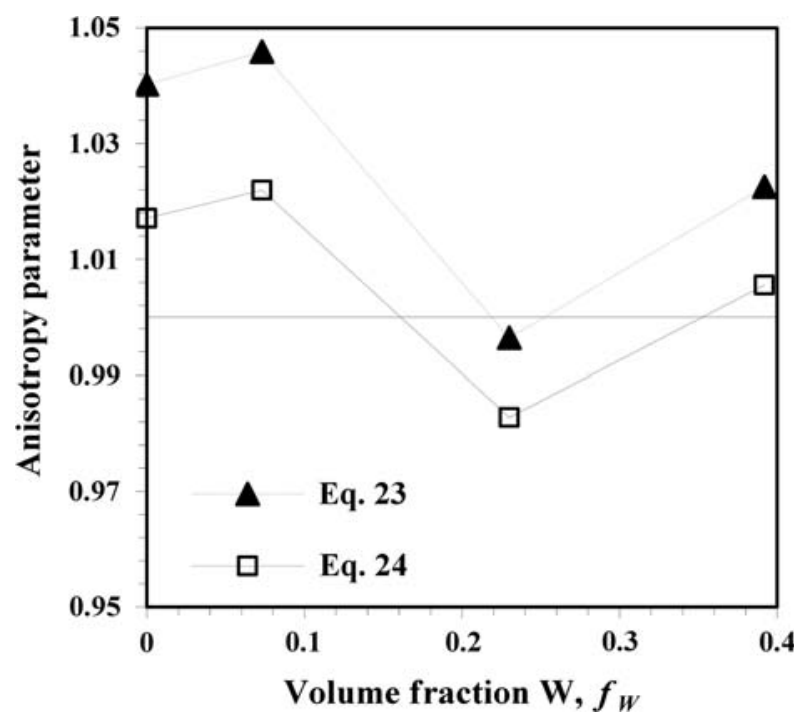

Fig. 7-Anisotropy parameters given by Eqs. [23] and [24] are plotted vs $f_{W}$. Complete isotropy corresponds to a value of 1.00 , and is indicated by the solid line for reference purposes. (i.e., $\left.\quad V_{12}=V_{13}=V_{31}=V_{23}=\ldots\right) \quad$ velocities, respectively. Figures 8 (a) and (b) show these ultrasonically determined moduli, along with $C_{33}$, plotted vs $f_{W}$. The $f_{p}$ is given for each composition by the labels next to the symbols for the shear modulus $G$. Again, the overall behavior of the elastic moduli shows the competing effects of stiffening associated with increased $f_{W}$ and softening associated with $f_{p}$. The elastic moduli shown in Figures 8(a) and (b) were evaluated within the context of the MT effective field model. ${ }^{[15,43,44]}$ The MT model has been applied to the estimation of the elastic properties of various two-phase composites. ${ }^{[43,45,46]}$ The approach approximates the effective elastic properties by assuming that an inclusion is subject to a uniform effective stress field. The model can be used to treat the case of porosity in a solid matrix by taking the moduli of the inclusion to be zero. For randomly oriented, spheroidal voids, the MT model yields the following relations for the bulk and shear moduli: ${ }^{[43,44]}$

$$
\begin{aligned}
& B=\frac{B_{o}}{1+p\left(S_{i j k l}\right) f_{W}} \\
& G=\frac{G_{o}}{1+q\left(S_{i j k l}\right) f_{W}}
\end{aligned}
$$

where $B_{o}$ and $G_{o}$ are the bulk and shear moduli of the matrix, respectively, in the absence of any inclusion phase. The terms $p\left(S_{i j k l}\right)$ and $q\left(S_{i j k l}\right)$ are functions of the components of the Eshelby stress tensor, $S_{i j k l}[47,48]$ The Eshelby stress tensor was introduced as part of a closed-form solution to the strain field within an elastic ellipsoidal inclusion in an elastic matrix subject to a uniform strain at infinity. The terms of $S_{i j k l}$ are, in turn, dependent upon the aspect ratio $\alpha$ of the inclusion via the secondary function $g(\alpha)$. For oblate spheroids:

$$
g(\alpha)=\alpha \frac{\left[\cos ^{-1}(\alpha)-\alpha\left(1-\alpha^{2}\right)^{1 / 2}\right]}{\left(1-\alpha^{2}\right)^{3 / 2}}
$$

Treatment of the present three-phase composites (Bi, W, and porosity) was performed as follows. The pore-free moduli ( $B$ and $G$ ) were calculated at each $f_{W}$ using Eqs. [28] through [30], and the moduli for Bi and $\mathrm{W}$ from Table I, by assuming $\alpha=1$ for the $\mathrm{W}$ inclusions. This aspect ratio corresponds to spherical inclusions, which is qualitatively consistent with the morphology of the W particles seen during microscopy. Then, in a second step, each $f_{W}$ was treated as an effective matrix with elastic moduli equal to the calculated pore-free moduli, and the porosity was treated as a second phase with moduli equal to zero. The aspect ratio of the porosity was then the only adjustable parameter available to fit the model to the data. After a few iterations by trial and error, $\alpha=0.1$ was found to give a suitable fit over most of the composition range. The results are shown as the dotted lines in Figures 8(a) and (b). The deviation of the data from the model predictions at $f_{W}=0.39$ is probably related to the percolation of the $\mathrm{W}$ powder. Note that 


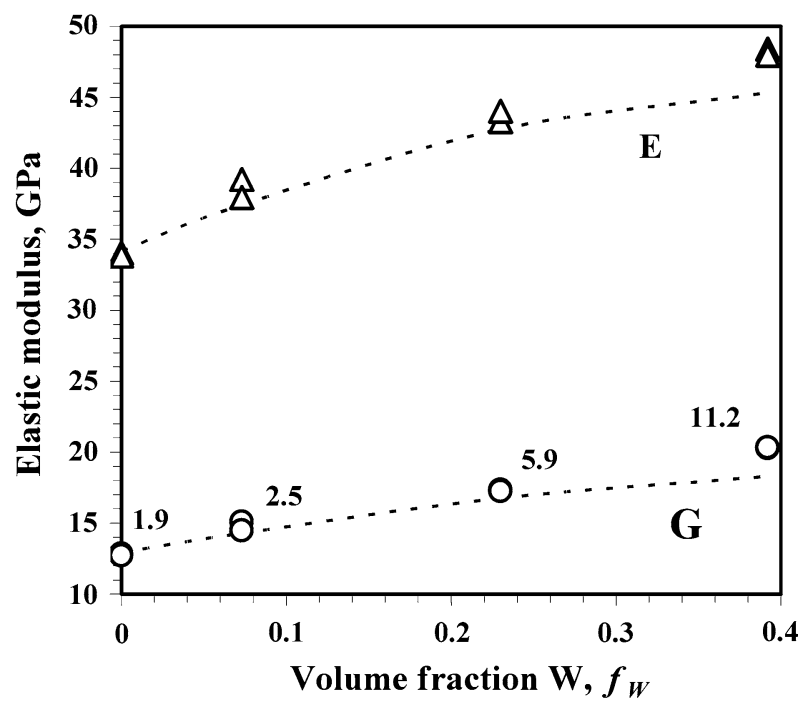

(a)

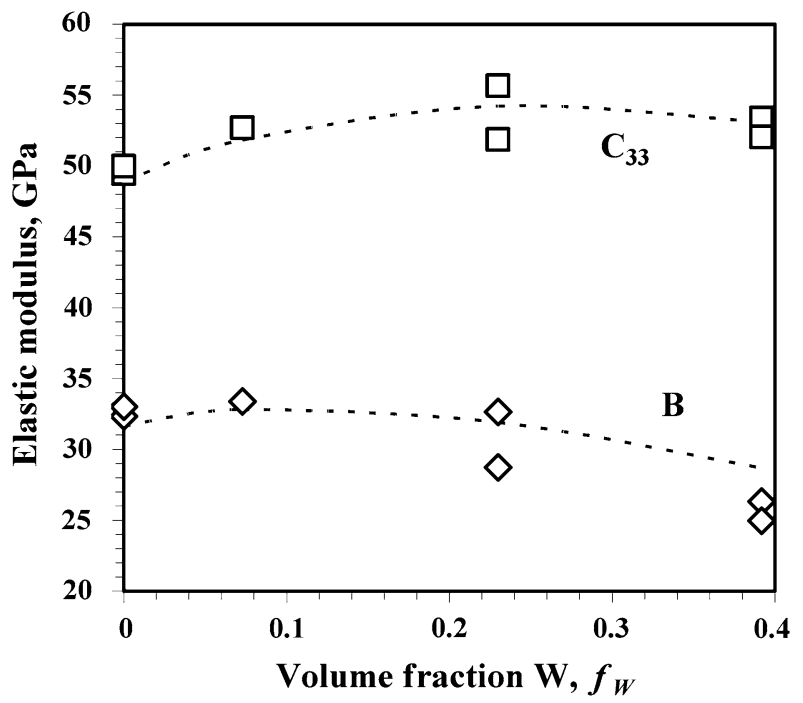

(b)

Fig. 8- (a) Engineering elastic moduli $(E, G$, and $B)$ calculated from the sound-wave velocities $V_{3}$ and $V_{31}$ using Eqs. [25] through [27]. (b) Also shown are ex-situ $C_{33}$ from Fig. 5. Values are plotted vs $f_{W}$, and the related $f_{p}$ are indicated by the labels next to the symbols for the shear modulus $G$. Dotted lines indicate moduli predicted by the MT model, assuming the aspect ratios of the $\mathrm{W}$ particles and the pores are $\alpha=1$ and 0.1 , respectively.

the reported aspect ratio is not meant to be exact, but rather to show qualitative behavior. That is, the analysis is not meant to imply that the pores are actually oblate spheroids with a single, well-defined aspect ratio. Rather, it is meant to qualitatively describe the behavior of the entire system of porosity as having an effect on the elastic moduli, which is equivalent to the isomorphous $(\alpha=0.1)$ spheroids.

Figure 9 shows the Vicker's hardness $H_{V}$ vs $f_{W}$ measured in both the axial (pressing) and radial (normal) directions for one series of samples. The error bars represent the standard deviation of the 10 measurements performed on each sample/direction. The data show increasing hardness with $f_{W}$, despite the concurrent

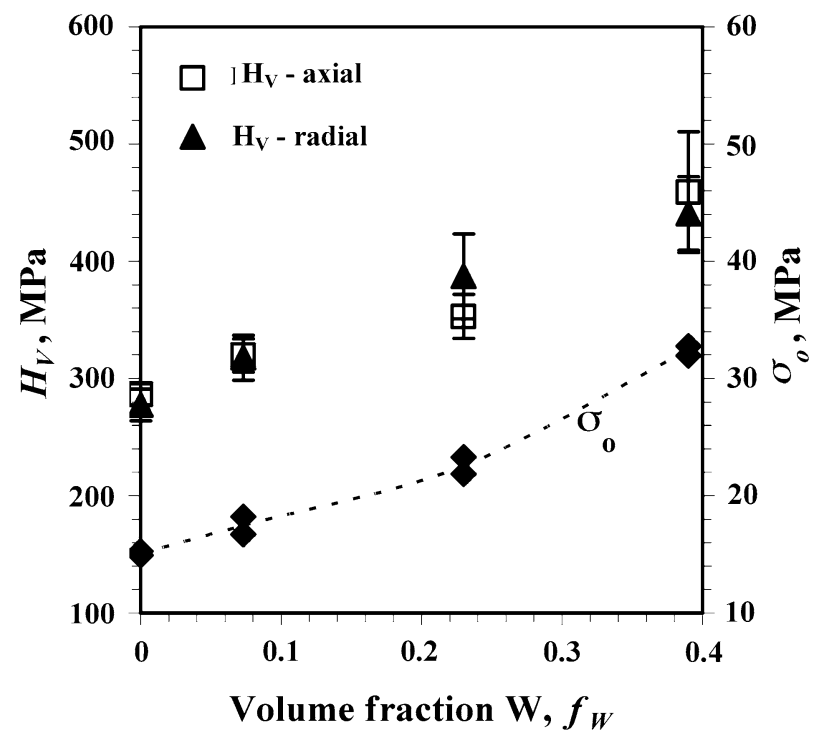

Fig. 9-Vicker's hardness measured in axial $\left(x_{3}\right)$ and radial $\left(x_{1}=x_{2}\right)$ directions $v s f_{W}$. Also shown is the pore-free yield strength $\sigma_{o}$ calculated by fitting Eq. [8] to the compaction data (Table II).

increase in $f_{p}$, ranging from $\sim 280 \mathrm{MPa}$ for $f_{W}=0$ to $\sim 480 \mathrm{MPa}$ for $f_{W}=0.39$. The increased scatter with increasing $f_{W}$ is related to the increasing heterogeneity in the microstructure as the $f_{W}$ and $f_{p}$ increase. That is, at the higher $f_{W}$, there is an increased probability that some of the indents will impinge on a pore or a $\mathrm{W}$ particle (or agglomerate), thus perturbing the measurement. Due to the multiphase nature of the composite, it is difficult to draw a direct correlation between the yield strength and the measured hardness. It has been observed that the yield strength is often proportional to the hardness and that, in the absence of strain hardening, the constant of proportionality is $\sim 1 / 3{ }^{[49]}$ Clearly, comparison of the measured $H_{V}$ at $f_{W}=0$ with the yield strengths reported for compression testing of $\mathrm{Bi}$ discussed earlier ${ }^{[24]}$ implies that this relation is not met for the current system. However, $\mathrm{Bi}$ is known to exhibit extensive work hardening, ${ }^{[24]}$ and the powder compaction process probably leaves the Bi in a highly cold-worked condition. Thus, the differences in the specimen fabrication methods may make direct comparison of the hardness with the prior compression data questionable.

The calculated $\sigma_{o}$ values determined by fitting Eq. [8] to the compaction data are plotted against the righthand axis in Figure 9. The calculated values exhibit qualitatively the same trend as the measures of hardness. This seems to confirm that the application of Eq. [8] to the compaction data provides at least a reasonable measure of the relative effect of $f_{W}$ on the strength. Careful inspection of the data shows that $\sigma_{o}$ actually increases slightly more rapidly with $f_{W}$ than does $H_{V}$; however, this is a reasonable and expected consequence of the residual porosity present in the samples during measurement of the hardness. Recall that $\sigma_{o}$ is defined as the expected yield strength in the fully dense material (Eq. [6]). 


\section{CONCLUSIONS}

This investigation examines the compaction behavior of composite mixtures of $\mathrm{Bi}$ and $\mathrm{W}$ powders via in-situ measurement of the thickness and sound-wave transit time during incremental compaction. The compaction behavior is evaluated using a modified Heckel equation, and the ultrasonically determined moduli are analyzed within the context of a site percolation model. Postcompaction analysis of the microstructure, sound-wave velocity, and Vicker's hardness was performed in order to further understand the mechanical properties and the effects of different fractions of $\mathrm{W}$ reinforcement and residual porosity $\left(f_{W}\right.$ and $\left.f_{p}\right)$. The results of the investigation can be summarized as follows.

1. The unreinforced Bi powder compacts to $\sim 98 \mathrm{pct}$ density at $540 \mathrm{MPa}$. The $\mathrm{W}$ powder inhibits the densification process, and this effect is most prominent at lower compaction pressures.

2. The modified Heckel equation describes the compaction behavior accurately. The fitting parameters (particularly $\sigma_{o}$ ) are sensitive to how the initial porosity is defined. The predicted yield strength $\sigma_{o}$ for $f_{W}=0$ is consistent with reported compression testing of pure $\mathrm{Bi}$, and increases with $f_{W}$.

3. A percolation model describes the behavior of the elastic modulus $C_{33}$ relative to the pore fraction from $f_{p}>0.4$ to $f_{p}<0.02$. The predicted pore-free $C_{33, o}$ fall within the theoretical bounds predicted by Hashin and Shtrikman.

4. While the microstructure shows some slight evidence of anisotropy between the axial and radial directions, a comparison of the ultrasonic velocities and Vicker's hardness shows the anisotropy to be $<5$ pct.

5. The engineering elastic moduli $(G, B$, and $E$ ) were calculated from the sound-wave velocities based on the assumption of complete isotropy. The effects of $f_{W}$ and $f_{p}$ on these elastic moduli were evaluated using the MT effective field model.

6. The measured Vicker's hardness increases with $f_{W}$. No direct correlation can be made between the $H_{V}$ and the yield strength at this time; however, $H_{V}$ and the $\sigma_{o}$ predicted from the Heckel equation exhibit qualitatively the same behavior.

\section{ACKNOWLEDGMENTS}

This work was performed under the auspices of the United States Department of Energy by the Lawrence Livermore National Laboratory (LLNL), under Contract No. DE-AC52-07NA27344. Project No. 06-SI005 was funded by the Laboratory Directed Research and Development Program at the LLNL.

\section{OPEN ACCESS}

This article is distributed under the terms of the Creative Commons Attribution Noncommercial
License which permits any noncommercial use, distribution, and reproduction in any medium, provided the original author(s) and source are credited.

\section{REFERENCES}

1. G.R. Odette, M.J. Alinger, and B.D. Wirth: Annu. Rev. Mater. Res., 2008, vol. 38, pp. 471-503.

2. R.W. Heckel: Trans. TMS-AIME, 1961, vol. 221, pp. 1001-08.

3. V.P. Bondarenko, S.S Dzhamarov, and A.A Baidenko: Powder Metall. Met. Ceram., 1974, vol. 13, pp. 12-15.

4. I. Shapiro: Adv. Powder. Metall., 1995, vol. 1, pp. 2.97-2.114.

5. P.J. Denny: Powder Metall., 2002, vol. 127, pp. 162-72.

6. R.J. Roberts and R.C. Rowe: Chem. Eng. Sci., 1987, vol. 42, pp. 903-11.

7. A.R. Boccaccini and Z. Fan: Ceram. Int., 1997, vol. 23, pp. 23945.

8. A.H. Tavakoli, A. Simchi, and S.S.S. Reihani: Comput. Sci. Technol., 2005, vol. 65, pp. 2094-2104.

9. C.-Y. Huang and G.S. Daehn: Acta Mater., 1996, vol. 44, pp. $1035-45$.

10. F.F. Lange, L. Atteraas, F. Zok, and J.R. Porter: Acta Metall. Mater., 1991, vol. 39, pp. 209-19.

11. C.D. Turner and M.F. Ashby: Acta Mater., 1996, vol. 44, pp. $4521-30$.

12. K.T. Kim, J.H. Cho, and J.S. Kim: J. Eng. Mater. Technol., 2000, vol. 122, pp. 119-28.

13. A.G. Yurshenko, L.I. Pugina, and N.I. Shcherban: Powder Metall. Met. Ceram., 1969, vol. 8, pp. 355-58.

14. Z. Hashin and S. Shtrikman: J. Mech. Phys. Solids, 1963, vol. 11, pp. $127-40$.

15. T. Mori and K. Tanaka: Acta Metall., 1973, vol. 21, pp. 571-74.

16. Performed via X-ray sedigraph per ASTM C-958-92.

17. L.P. Martin, A.M. Hodge, and G.H. Campbell: Scripta Mater., 2007, vol. 57, pp. 229-32.

18. K.T. Kim, S.C. Lee, and H.S. Ryu: Mater. Sci. Eng., A, 2003, vol. A340, pp. 41-48.

19. I. Shapiro and I.M. Kolthoff: J. Phys. Colloid Chem., 1947, vol. 51, pp. 483-93.

20. K. Konopicky: Radex-Rundschau, 1948, vol. 3, pp. 141-48.

21. R.W. Heckel: Trans. TMS-AIME, 1961, vol. 221, pp. 671-75.

22. M. Kuentz and H. Leuenberger: J. Pharm. Sci., 1999, vol. 88, pp. 174-79.

23. R. Leu and H. Leuenberger: Int. J. Pharm., 1993, vol. 90, pp. 213 19.

24. G.C. Das and M.B. Bever: Metall. Trans. A, 1978, vol. 9A, pp. $1875-81$.

25. L.P. Martin, D. Dadon, and M. Rosen: J. Am. Ceram. Soc., 1996, vol. 79 , pp. $1281-89$.

26. E.P. Papadakis and B.W. Peterson: Mater. Eval., 1979, vol. 37, pp. $76-80$

27. D.J. Roth, D.B. Stang, S.M. Swickard, M.R. DeGuire, and L.E. Dolhert: Mater. Eval., 1991, vol. 49, pp. 883-88.

28. O. Yeheskel, M. Shokhat, M. Ratzker, and M.P. Dariel: J. Mater. Sci., 2001, vol. 36, pp. 1219-25.

29. R.M. Spriggs: J. Am. Ceram. Soc., 1961, vol. 44, pp. 628-29.

30. K.K. Phani and S.K. Niyogi: J. Mater. Sci., 1987, vol. 22, pp. $257-$ 63.

31. R.L. Coble and W.D. Kingery: J. Am. Ceram. Soc., 1956, vol. 39, pp. $377-85$.

32. J. Kovacik: J. Mater. Sci. Lett., 1999, vol. 18, pp. 1007-10.

33. D. Stauffer: Introduction to Percolation Theory, Taylor and Francis, London, 1985.

34. I. Carabello, M. Fernandez-Arevalo, M. Milan, A.M. Rabasco, and H. Leuenberger: Int. J. Pharm., 1996, vol. 139, pp. 177-86.

35. B. van Veen, K. van der Voort Maarschalk, G.K. Bolhuis, and H.W. Frijlink: Powder Technol., 2004, vol. 139, pp. 156-64.

36. C.-W. Nan and D.M Smith: Mater. Sci. Eng., 1991, vol. B10, pp. L1-L3.

37. J. Kovacik: J. Mater. Sci. Lett., 2001, vol. 20, pp. 1953-55.

38. H. Leuenberger and L. Ineichen: Eur. J. Pharm. Biopharm., 1997, vol. 44, pp. 269-72. 
39. L.E. Holman and H. Leuenberger: Powder Technol., 1991, vol. 64, pp. 233-47.

40. Z. Hashin: J. Appl. Mech., Trans. ASME, 1962, vol. 29, pp. 143-50.

41. M.L. Hentschel and N.W. Page: J. Mater. Sci., 2007, vol. 42, pp. 1269-78.

42. I. Song, M. Suh, Y.-K. Woo, and T. Hao: Eng. Geol., 2004, vol. 72, pp. 293-308.

43. Y.H. Zhao, G.P. Tandon, and G.J. Weng: Acta Mech., 1989 , vol. 76, pp. 105-30.

44. G.P. Tandon and G.J. Weng: Compos. Sci. Technol., 1986, vol. 27, pp. 111-32.
45. H. Ledbetter, M. Dunn, S. Kim, and R. Fields: in Review of Quantitative NDE, D.O. Thompson and D.E. Chimenti, eds., Plenum Press, New York, NY, 1995, vol. 14, pp. 1633-39.

46. J.B. Walsh, W.F. Brace, and A.W. England: J. Am. Ceram. Soc., 1965, vol. 48, pp. 605-08.

47. T.T. Wu: Int. J. Solids Struct., 1966, vol. 2, pp. 1-8.

48. J.D. Eshelby: Proc. R. Soc. London, Ser. A, 1959, vol. 252, pp. 561-69.

49. J.R. Cahoon, W.H. Broughton, and A.R. Kutzak: Metall. Trans., 1971, vol. 2, pp. 1979-83. 